\title{
Institutional Review Boards and Bioethical Issues for Otologists and Audiologists
}

\author{
Moo Kyun Park and Byung Don Lee \\ Department of Otorhinolayngology-Head and Neck Surgery, Bucheon Hospital, Soonchunhyang University College of Medicine, \\ Bucheon, Korea
}

Received June 20,2012

Revised July 2, 2012

Accepted July 3, 2012

Address for correspondence

Moo Kyun Park, MD, PhD

Department of Otorhinolayngology-

Head and Neck Surgery,

Bucheon Hospital,

Soonchunhyang University

College of Medicine,

170 Jomaru-ro, Wonmi-gu,

Bucheon 420-767, Korea

Tel +82-32-621-5054

Fax +82-32-621-5440

E-mail aseptic@schmc.ac.kr
Otologists and audiologists care for patients and conduct clinical research to find more effective treatments that benefit patients. Institutional Review Board (IRB) permission is necessary for conducting clinical trials on humans. Furthermore, many bioethical conflicts are encountered while conducting research. However, few otologists and audiologists in Korea know bioethics and the principles and regulations of IRBs in detail. This paper reviews the history of ethics in clinical research and current bioethical principles and IRB regulations. We outline what you need as otologists or audiologists to get IRB approval while considering the principles of bioethics.

Korean J Audiol 2012;16:43-46

KEY WORDS: Institutional review boards · Bioethics · Research · Otologist.

\section{Introduction}

Clinical research starts with medical practice. There is evidence that ancient Egyptian, Greek, and Chinese physicians conducted clinical studies, including studies of drugs, surgery, and devices. ${ }^{1)}$ Hundreds of studies related to hearing have been conducted in the USA. In Korea, over 100 studies related to hearing or otology are published annually in national or international journals. Clinical research is not limited to universities or tertiary hospitals. Most otologists and audiologists know that Institutional Review Board (IRB) permission is essential when conducting clinical trials involving humans. Many ethical issues arise in clinical practice and clinical research. As physicians and researchers, we often care for patients and conduct clinical research simultaneously. ${ }^{2)}$ However, conflict between clinical practice and research can arise. For example, a research participant may not gain any benefit from the research, but the research may have great benefit for others. When one finds a potentially good drug or treatment for a disease, one wants to test it. Therefore, one must know bioethics and IRB principles and regulations so as to get IRB approval to conduct clinical research. As with clinical guidelines and well-known treatment protocols, there are rules that must be followed. Additionally, governments may restrict clinical trials by law (Table 1).

\section{History of Clinical Research and Bioethics}

Clinical trials started with the beginning of medicine. Perhaps ethical issues were also a part of medicine at that time. The first chapter of Daniel in the Old Testament (Daniel 1: 11-16) contains evidence of a clinical trial, and Hippocrates tried to define ethical issues in medicine. However, ethical issues emerged as a major concern in clinical trials before World War II. As a result of Nazi experiments and other unethical experiments conducted in the $20^{\text {th }}$ century, codes and regulations have been drawn up to protect human subjects (Table 1). ${ }^{3-8)}$ The judgment at Nuremberg with respect to the Nazi experiments identified two essential ethical points: voluntary consent and the rights or welfare of the subject (Table 2). ${ }^{1,3)}$

The Declaration of Helsinki, originally issued by the World Medical Assembly in 1964, has been revised seven times as of 2011. ${ }^{6.9)}$ The guiding principle is "the health of my patient will 
be my first consideration". Although the declaration applies primarily to physicians, the World Medical Assembly encouraged those conducting related medical research to follow their principles. They defined several requirements for the scientific and ethical aspects of research to be applied by physicians, authors, and ethics committees. ${ }^{6}$

\section{Principles of Bioethics}

There are several principles in bioethics. The three basic principles identified in the Belmont report in 1979 were respect for persons, beneficence, and justice. These principles are manifested in informed consent, the assessment of risk and benefits, and the selection of subjects. ${ }^{10)}$

The seven ethical requirements published in the Journal of the American Medical Association in 2000 are also wellknown and well-organized principles (Table 3). ${ }^{11)}$ Fulfillment of all seven requirements is necessary for ethical clinical research. ${ }^{11,12)}$ These requirements are universal, but they must be adapted to the health, economic, cultural, and technological conditions under which clinical research is conducted.

Clinical trials are valuable if the clinical research improves

Table 1. Codes and regulations for clinical research

The Nuremberg code (1949)

The Belmont Report (1979)

ICH GCP [internal conference on harmonization guidelines for good clinical practice (1996)]

CIMOS (Council for International Organizations of Medical

Sciences)

International Ethical Guidelines for Biomedical Research (2002)

The Declaration of Helsinki (2011)

Bioethics and Safety Act (act number 9932, enforcement date 18, Jan, 2010) health and well-being or advances knowledge. To be ethical, clinical research must be valuable. All clinical research exploits patients and uses limited resources. So investigators should consider the value of any clinical trial. ${ }^{13)}$

Scientific validity means that the researcher should use accepted scientific principles and methods, including statistical methods, to produce reliable, valid data. ${ }^{4}$ In clinical research, a scientific study may be an ethical study; an unscientific study is an unethical study. Subject selection must be fair so that stigmatized or vulnerable subjects are not targeted for risky research. A scientific study requires fair subject selection using scientifically valid selection and exclusion criteria. Through this process, the investigator can manage risks and benefits, such as excluding higher-risk groups. ${ }^{14,15)}$

Clinical trials can be conducted only if they have a favorable risk-benefit ratio. ${ }^{16)}$ Risk is the probability of harm or injury caused by the clinical trial. Minimal risk is defined as "the probability and magnitude of harm or discomfort anticipated in the research are not greater in and of themselves than those ordinarily encountered in daily life or during the performance of routine physical or psychological examinations or tests" [Code of Federal Regulations (CFR) 45, 46]. Benefit refers to valued or desired outcomes related to health, psychosocial state, or knowledge. Money is not a benefit, but can be considered as compensation. ${ }^{17)}$ The assessment of risk and bene-

Table 3. The seven ethical requirements
1. Value
2. Scientific validity
3. Fair subject selection
4. Favorable risk-benefit ratio
5. Independent review
6. Informed consent
7. Respect for enrolled subjects

Table 2. Nuremberg codes $^{7)}$

1. The voluntary consent of the human subject is absolutely essential.

2. The experiment should be such as to yield fruitful results for the good of society, unprocurable by other methods or means of study, and not random and unnecessary in nature.

3. The experiment should be so designed and based on the results of animal experimentation and a knowledge of the natural history of the disease or other problem under study that the anticipated results will justify the performance of the experiment.

4. The experiment should be so conducted as to avoid all unnecessary physical and mental suffering and injury.

5. No experiment should be conducted where there is an a priori reason to believe that death or disabling injury will occur; except, perhaps, in those experiments where the experimental physicians also serve as subjects.

6. The degree of risk to be taken should never exceed that determined by the humanitarian importance of the problem to be solved by the experiment.

7. Proper preparations should be made and adequate facilities provided to protect the experimental subject against even remote possibilities of injury, disability, or death.

8. The experiment should be conducted only by scientifically qualified persons. The highest degree of skill and care should be required through all stages of the experiment of those who conduct or engage in the experiment.

9. During the course of the experiment the human subject should be at liberty to bring the experiment to an end if he has reached the physical or mental state where continuation of the experiment seems to him to be impossible.

10. During the course of the experiment the scientist in charge must be prepared to terminate the experiment at any stage, if he has probable cause to believe, in the exercise of the good faith, superior skill and careful judgment required of him that a continuation of the experiment is likely to result in injury, disability, or death to the experimental subject. 
fits involves three steps. First, risks are identified and minimized. Risk can be reduced by using a scientific study design and reducing unnecessary exposure to risk. ${ }^{18,19)}$ Second, benefits are increased if the health of the subjects is improved, knowledge about their disease is increased, or a clinical service is provided. Finally, the balance between risks and benefit should be assessed. The cumulative benefit of a clinical trial should outweigh its risk.

Independent review is essential when conducting clinical trials because every investigator has an inherent conflict of interest. $^{20)}$ The independent reviewers should not be affiliated with the research to minimize any conflict of interest. ${ }^{21,22)}$ The independent review includes not only the IRB but also dataand safety-monitoring boards or ethics committees. ${ }^{23)}$ An independent reviewer reviews the design of the research, the proposed subject population, and the risk-benefit ratio of the study. Minimum standards have been defined for IRB membership: the IRB should have at least five members including at least one member with a scientific background, one nonscientific member, and one person who is not affiliated with the institution. ${ }^{5)}$

The investigator should respect subject autonomy and should obtain informed consent. ${ }^{24)}$ Informed consent must ensure that individuals control their enrollment in clinical trials and their continuing enrollment. Any informed consent process should include notifying the subject of the purpose of the re- search as well as its procedures, potential risks, benefits, and alternatives. In the USA, the general requirements for informed consent are regulated by the CFR (Table 4). ${ }^{5)}$

\section{IRB Review Process}

The aim of most IRBs is to protect the rights and welfare of humans participating as subjects in research studies. The IRB approves and monitors clinical trials. Although the IRB review process varies according to region and institution, it includes common steps and rules. ${ }^{13,25)}$ The process starts with the submission of a research proposal. The proposal usually includes the application form, study protocol, case report form, curriculum vitae of the investigators, and statement of conflicts of interest. Additionally, most IRBs require the completion of good clinical practice training, Collaborative Institutional Training Initiative training, or other training to ensure that the investigators are prepared to conduct ethical research.

When the proposal is submitted, the IRB reviews it in terms of its scientific and ethical merit. ${ }^{26)}$ In the USA, CFR 46 suggests that regulatory review is a requirement (Table 5). ${ }^{5)}$ After the review, the protocol is voted on; the vote may be open or closed. Four basic outcomes are possible: approval, conditional approval, revision required, and rejection. After obtaining IRB approval, clinicians can start their studies. Typically, the IRB requires that the approval be renewed annually if the stu-

Table 4. Basic elements of informed consent (CFR 45, 46.116)

1) A statement that the study involves research, an explanation of the purposes of the research and the expected duration of the subject's participation, a description of the procedures to be followed, and identification of any procedures which are experimental;

2) A description of any reasonably foreseeable risks or discomforts to the subject;

3) A description of any benefits to the subject or to others which may reasonably be expected from the research;

4) A disclosure of appropriate alternative procedures or courses of treatment, if any, that might be advantageous to the subject;

5) A statement describing the extent, if any, to which confidentiality of records identifying the subject will be maintained;

6) For research involving more than minimal risk, an explanation as to whether any compensation and an explanation as to whether any medical treatments are available if injury occurs and, if so, what they consist of, or where further information may be obtained;

7) An explanation of whom to contact for answers to pertinent questions about the research and research subjects' rights, and whom to contact in the event of a research-related injury to the subject; and

8) A statement that participation is voluntary, refusal to participate will involve no penalty or loss of benefits to which the subject is otherwise entitled, and the subject may discontinue participation at any time without penalty or loss of benefits to which the subject is otherwise entitled.

CFR, US Code of Federal Regulations

Table 5. Regulatory review requirements (45 CFR 46.111)
1) Risks to subjects are minimized
2) Risks to subjects are reasonable in relation to anticipated benefits
3) Selection of subjects is equitable
4) Informed consent will be sought from each prospective subject or the subject's legally authorized representative
5) Informed consent will be appropriately documented
6) The research plan makes adequate provision for monitoring the data collected to ensure the safety of subjects
7) There are adequate provisions to protect the privacy of subjects and to maintain the confidentiality of data

CFR, US Code of Federal Regulations 
dy will continue for several years.

\section{Conclusions}

Before commencing clinical research, it is important to know bioethics as well as the IRB rules. As otologists and audiologists, we can reduce the risk to participants and provide more information for informed consent in studies involving hearingimpaired patients. Any well-designed study protocol should meet scientific and ethical guidelines.

\section{Acknowledgments}

The author thanks Dr. Christine Grady and Dr. Marion Danis of $\mathrm{NIH}$ for providing the bioethics research data.

\section{REFERENCES}

1) Gallin JI. A historical perspective on clinical research. In: Gallin JI, Ognibene FP, editors. Principles and practice of clinical research. Burlington: Elsevier;2007. p.1-10.

2) Beecher HK. Ethics and clinical research. N Engl J Med 1966;274: 1354-60.

3) Nuremberg Military Tribunal. The Nuremberg Code. JAMA 1996; 276:1691.

4) Sciences CfIOoM. International Ethical Guidelines for Biomedica Research Involving Human Subjects. Geneva, CIOMS/WHO;2002 [cited 2002]; Available from: URL: http://www.cimos.ch.

5) Huisman MA, De Heer E, Grote JJ. Survival signaling and terminal differentiation in cholesteatoma epithelium. Acta Otolaryngol 2007; 127:424-9.

6) Chicheportiche Y, Bourdon PR, Xu H, Hsu YM, Scott H, Hession C, et al. TWEAK, a new secreted ligand in the tumor necrosis factor family that weakly induces apoptosis. J Biol Chem 1997;272:3240110

7) Albino AP, Reed JA, Bogdany JK, Sassoon J, Desloge RB, Parisier SC. Expression of p53 protein in human middle ear cholesteatomas: pathogenetic implications. Am J Otol 1998;19:30-6.

8) Council for International Organizations of Medical Sciences. International ethical guidelines for biomedical research involving human subjects. Bull Med Ethics 2002:17-23.
9) World Medical Association declaration of Helsinki. Recommendations guiding physicians in biomedical research involving human subjects. JAMA 1997;277:925-6.

10) Grady C. Ethical principles in clinical research. In: Gallin JI, Ognibene FP, editors. Principles and practice of clinical research. 2nd ed. Burlington: Elsevier;2007. p.15-25.

11) Emanuel EJ, Wendler D, Grady C. What makes clinical research ethical? JAMA 2000;283:2701-11.

12) Miller FG. Clinical research with healthy volunteers: an ethical framework. J Investig Med 2003;51 Suppl 1:S2-5.

13) Danis M, Largent E, Grady C, Wendler D, Hull SC, Shah S, et al. Starting research. New York: Oxford University Press;2012. p.21-48.

14) Miller J, Crigger BJ. Ethical standards for human subject research in developing countries. IRB 1992;14:7-8.

15) Arpaillange P, Dion S, Mathe G. Proposal for ethical standards in therapeutic trials. Br Med J (Clin Res Ed) 1985;291:887-9.

16) Kaufman JL. Protecting research subjects. N Engl J Med 2002;346: 2093-5; author reply 2093-5.

17) Bernstein M. Payment of research subjects involved in clinical trials is unethical. J Neurooncol 2003;63:223-4.

18) Danis M, Largent E, Grady C, Wendler D, Hull SC, Shah S, et al. Enrolling research participants. New York: Oxford University Press; 2012. p.49-77.

19) Danis M, Largent E, Grady C, Wendler D, Hull SC, Shah S, et al. Protecting research participants. New York: Oxford University Press; 2012. p.78-99.

20) Kaiser J. Clinical trials. Proposed rules aim to curb financial conflicts of interest. Science 2002;295:246-7.

21) American Society of Clinical Oncology. American Society of Clinical Oncology policy statement: oversight of clinical research. J Clin Oncol 2003;21:2377-86.

22) Greco D, Diniz NM. Conflicts of interest in research involving human beings. J Int Bioethique 2008;19:143-54, 202-3.

23) Hossne WS, Vieira S, De Freitas CB. Committees for Ethics in Research involving human subjects. J Int Bioethique 2008;19:131-41, 200.

24) Bratt DE. Informed consent in medical research. Doctors are arrogant to think they need to debate issue of patient consent. BMJ 1997; 314:1477.

25) Wichman A. Institutional review boards. In: Gallin JI, Ognibene FP, editors. Principles and practice of clinical research. Burlington: Elsevier;2007. p.47-57.

26) Weymuller EA Jr. A consideration of ethical issues in the design of clinical trials. Am J Otolaryngol 1996;17:2-11. 